\title{
Diagnostic and Prognostic Significance of Dysregulated Expression of Circular RNAs in Osteosarcoma
}

\author{
Chenghao Zhang \\ The Second Xiangya Hospital, Central South University, China \\ Jieyu $\mathrm{He}$ \\ The Second Xiangya Hospital, Central South University, China \\ Lin Qi \\ The Second Xiangya Hospital, Central South University, China \\ Zhixi Duan \\ The Second Xiangya Hospital, Central South University, China \\ Lu Wan \\ The Second Xiangya Hospital, Central South University, China \\ Wanchun Wang \\ The Second Xiangya Hospital, Central South University, China \\ Chao Tu ( $\nabla$ tuchao@csu.edu.cn ) \\ https://orcid.org/0000-0001-8267-4727 \\ Zhihong Li \\ The Second Xiangya Hospital, Central South University, China
}

\section{Research article}

Keywords: CircRNA, osteosarcoma, diagnosis, prognosis, survival

Posted Date: June 24th, 2020

DOI: https://doi.org/10.21203/rs.3.rs-34455/v1

License: (c) (i) This work is licensed under a Creative Commons Attribution 4.0 International License. Read Full License

Version of Record: A version of this preprint was published on January 22nd, 2021. See the published version at https://doi.org/10.1080/14737159.2021.1874922. 


\section{Abstract \\ Background}

CircRNAs have emerged as pivotal regulators in osteosarcoma tumorigenesis and progression, but their prognostic and diagnostic significance remain unclear. Herein, we aimed to perform an updated meta-analysis to explore the clinical, diagnostic and prognostic values of circRNAs in osteosarcoma.

\section{Methods}

Several databases, including PubMed, Web of Science, EMBASE, Scopus and Cochrane Library, were systematically searched up to April $10,2020$. Eligible studies regarding the relationship between circRNAs levels and clinicopathological, diagnostic and prognostic values in osteosarcoma patients were included in this study. Pooled odds ratios with corresponding $95 \%$ confidence intervals were used to measure clinical characteristics, while hazard ratios with $95 \%$ Cls were adopted to assess overall survival (OS) and disease-free survival (DFS).

\section{Results}

Overall, 26 relevant studies involving 1,652 patients with osteosarcoma were enrolled, with eighteen studies on clinicopathological parameters, ten on diagnosis and eighteen on prognosis. For clinical parameters, overexpression of oncogenic circRNAs was intimately correlated with larger tumor size $(P<0.00001)$, advanced Enneking stage $(P<0.00001)$, poor differentiation $(P=0.0001)$, and distant metastasis $(D M)(P<0.00001)$. In contrast, the downregulated circRNAs showed negative correlation with Enneking stage $(P=0.002)$ and $D M(P<0.0001)$. For the diagnostic values, the summary area under the curve of circRNA for the discriminative efficacy between osteosarcoma patients and non-cancer counterparts was estimated to be 0.86 (95\% Cl: $0.83-0.89)$, with a weighted sensitivity of 0.80 (95\% Cl: $0.74-0.84)$, specificity of 0.80 (95\%: $0.75-0.84)$, and diagnostic odds ratio of 15.48 (10.85-22.10), respectively. For the prognostic significance, oncogenic circRNAs had poor OS (HR = 1.92, $95 \% \mathrm{Cl}$ : 1.68-2.19) and DFS (HR = 2.65, 95\% Cl: 2.02-3.49), while elevated expression of tumor-suppressor circRNAs were closely related to longer OS (HR $=0.44,95 \% \mathrm{Cl}: 0.28-0.69)$.

\section{Conclusions}

Taken together, our study showed that aberrantly expressed circRNA signatures could serve as potential biomarkers in diagnosis and prognosis in patients with osteosarcoma.

\section{Background}

Osteosarcoma is a common primary bone malignancy that mainly affected children and adolescents [1]. In spite of substantial achievement in diagnosis and treatment, the 5-year survival of osteosarcoma remains unsatisfied [2], largely due to the insufficiency of accurate predictive biomarkers [3]. Therefore, it is urgent to identify more ideal biomarkers with both diagnostic and prognostic value in osteosarcoma.

Recent advances in transcriptomics have provided novel perspective for management of cancer [4]. Circular RNAs (circRNAs) are a cluster of endogenous non-coding RNAs (ncRNAs) with no or limited capacity for coding proteins [5-8]. It is formed from back-splicing of pre-RNAs without 3'terminal poly A tail and 5'-terminal cap [9]. Accordingly, it is more stable than the linear mRNA due to the covalently continuous loop [10]. Mechanistically, circRNAs could function as nuclear transcription regulator, attaching elements of RNA-binding protein (RBP), or competing endogenous RNA (ceRNA) to sponge microRNA (miRNA), thus in turn to play a critical role in various biological process of human diseases including cancer [11]. Interestingly, several circRNAs have been found to encode target genes, and thereby serve as tumor promoter or suppressor in cancer progression $[7,8]$.

Nowadays, circRNAs have attracted the researches attention as potential biomarkers and therapeutic targets in carcinomas, which are attributed to their abundance in tissues, structural stability and tissue-specific expression profile [9]. More recently, emerging studies have highlighted the role of circRNAs in osteosarcoma [12-19]. CircRNAs are reported to modulate osteosarcoma malignant properties including cell proliferation, apoptosis, migration and resistance to chemotherapy $[12,20,21]$. CircRNAs could interact with cancer-related signaling pathway, such as Akt/GSK-3 $\beta$, and wnt/ $\beta$-catenin pathway, or act as a ceRNA to sponge miRNA and consequently regulate target genes in osteosarcoma progression [12, 22, 23]. Moreover, a growing number of evidences showed that dysregulated circRNAs are closely correlated with survival outcome and clinicopathological parameters including clinical stage, chemo-sensitivity, and metastasis [13, 23, 24]. However, the clinical prognostic and diagnostic significance of circRNAs may be jeopardized due to the controversial results as well as limited sample size in previous studies. The aim of this meta-analysis was to further clarify the pooled clinicopathological, diagnostic and prognostic significance of circRNAs in osteosarcoma. 


\section{Methods \\ Search strategy}

This study was performed in accordance with the Preferred Reporting Items for Systematic Review and Meta-analysis (PRISMA) Checklist [25]. Several electronic databases, including PubMed, Web of Science, EMBASE, and the Cochrane Library database, were extensively searched from inception to April 10, 2020 for eligible studies that assessed the clinicopathological, diagnostic or prognostic significance of circRNAs in osteosarcoma. The following terms were used in databases for literature retrieval: ("circular RNA" or "circRNA" or "hsa_circ") and (osteosarcoma). Patients with osteosarcoma were considered as the "case group", while those with benign lesions or healthy were taken as "control group".

\section{Study Selection Criteria And Data Extraction}

Studies were eligible if met the following criteria: 1) cohort or case-control study; 2) diagnosis of osteosarcoma was histo-pathologically confirmed; and 3) correlation between circRNAs expression with clinicopathological features, diagnostic accuracy or prognosis utility were provided or extractable;

Besides, those studies were excluded if 1 ) not relevant with circRNAs or osteosarcoma; 2 ) in vivo studies, case reports, reviews or letters without original data; 3) duplicate studies;

Two independent investigators ( $\mathrm{CHZ}$ and $\mathrm{JYH}$ ) evaluated the enrolled studies and extracted data carefully, and a third researcher (LQ) would be consulted to reach a consensus if disagreement occurred. The following data were extracted in eligible studies: first author, publication year, circRNA type, sample type, sample size, detection assay, reference gene, regulation pattern, area under curve (AUC), circRNA expression levels, survival outcomes including overall survival (OS), disease-free survival (DFS) or progression-free survival (PFS), survival analysis method, follow-up duration (months), age, gender, tumor size, Enneking stage, differentiation based on WHO grade, and distant metastasis (DM).

\section{Quality Assessment, Sensitivity Analysis And Publication Bias}

The quality of included studies on diagnosis was assessed according to the Quality Assessment for Studies of Diagnostic Accuracy II (QUADAS II) checklist [26], while studies on prognosis were rated by the Newcastle-Ottawa Scale (NOS) as previously described [27, 28]. The studies were considered of high quality if the QUADAS II score was $\geq 4$, or NOS score was $\geq 6$.

In order to increase the credibility of this study, sensitivity analysis was performed to identify the potential source of heterogeneity. Publication bias was investigated by using both funnels plots and Begg's as well as Egger's test.

\section{Statistical analysis}

Statistical analyses were performed by using STATA software (13.0) and Meta-Disc (1.4). Pooled odds ratios (ORs) with 95\% confidence intervals (Cls) were adopted for evaluation of clinical features, while hazard ratios (HRs) with $95 \%$ Cls to measure OS and DFS. The Chi-square test was utilized to assess the heterogeneity among studies. If $P<0.05$ or $P>50 \%$, the random-effect model would be used due to the significant heterogeneity. Otherwise, the fixed-effect model would be adopted in the analyses. In addition, the publication bias was conducted by Deeks' funnel plot asymmetry test, Begg's and Egger's test [29].

\section{Results}

\section{Characteristics of the enrolled studies}

The search procedure was presented in Fig. 1. Among the potential literature retrieved from databases, 170 studies were initially assessed. After removing 90 duplicate publications, the remaining 80 studies were evaluated for titles and abstracts. Of these, 32 irrelevant articles were further excluded after abstract review and only 48 studies remained for full-text verification. Moreover, 22 studies were further eliminated with a variety of reasons, including five studies not related to circRNAs or osteosarcoma, six studies did not report outcomes, two reviews and case reports, four animal studies, and five lack of extractable data. Finally, 26 studies comprising 1,652 osteosarcoma cases were enrolled in this quantitative study, with 18 on clinicopathological features, ten on diagnosis $[13,18,19,21,24,30-34]$ and 18 on prognosis [12-14, 16-20, 22-24, 30-32, 34-42]. Among the 1,652 eligible osteosarcoma cases, patients were separated by age, gender, or other clinicopathologic features. As for age, the included studies had divergent cut-off values, with six studies on upregulated circRNAs using $\geq 25$ or $<25$ years old $[12,14,18,19,32-34,36-40]$, whereas other two studies on downregulated circRNAs applying $\geq 18$ or $<18$ years old to separate enrolled patients [13,30]. In consideration of the consistency, only these studies with common cut-off value were included in the following meta-analysis with regard to patients' age. Besides, all cases in studies on clinicopathological feature analysis were divided into male and female. Other clinicopathologic parameters consist of tumor 
size ( $\geq 5 \mathrm{~cm} /<5 \mathrm{~cm}$ ), Enneking stage (IIB-III/I-IIA), distant metastasis (positive/negative), and WHO grade (III/I-II) were also presented in this analysis.

The baseline characteristic features of enrolled studies were shown in detail in Table 1 and Table 2. All studies were conducted in China, and the publication year ranged from 2017-2020. All osteosarcoma cases were reliably diagnosed based on histopathology. The sample size was varied from 30 to 170. CircRNAs expression was measured by quantitative real-time polymerase chain reaction (qRT-PCR) and the reference gene was $G A P D H$. CircRNAs were regarded as oncogenes or tumor-suppressor in terms of their expression levels. Specifically, 21 circRNAs were identified as oncogenic circRNAs with upregulated expression pattern [12, 14, 16-23, 31-34, 36, 37, 39-43], while the other five were recognized as tumor suppressors $[13,24,30,35,38]$ with downregulated expression, respectively. The duration for follow-up ranged from 40 to 125 . For diagnostic assay, ten studies with data on sensitivity, specificity and AUC [13, 18, 19, 21, 24, 30-34].

Table 1

Main characteristics of studies for diagnosis analysis in osteosarcoma

\begin{tabular}{|c|c|c|c|c|c|c|c|c|c|c|}
\hline \multirow[t]{2}{*}{ Study } & \multirow{2}{*}{$\begin{array}{l}\text { Publication } \\
\text { year }\end{array}$} & \multirow[t]{2}{*}{ CircRNA } & \multirow{2}{*}{$\begin{array}{l}\text { Sample } \\
\text { type }\end{array}$} & \multicolumn{2}{|c|}{ Sample size } & \multirow[t]{2}{*}{ Method } & \multirow{2}{*}{$\begin{array}{l}\text { Reference } \\
\text { gene }\end{array}$} & \multirow{2}{*}{$\begin{array}{l}\text { Regulation } \\
\text { pattern }\end{array}$} & \multirow[t]{2}{*}{ AUC } & \multirow[t]{2}{*}{ Ref. } \\
\hline & & & & Case & Control & & & & & \\
\hline $\mathrm{Li}, \mathrm{S}$ et al & 2020 & circ_0000190 & serum & 60 & 60 & $\begin{array}{l}\text { qRT- } \\
\text { PCR }\end{array}$ & GAPDH & downregulated & 0.889 & [30] \\
\hline $\mathrm{Hu}, \mathrm{Y}$ et al & 2019 & circ-LARP4 & tissue & 72 & 72 & $\begin{array}{l}\text { qRT- } \\
\text { PCR }\end{array}$ & $G A P D H$ & downregulated & 0.829 & [13] \\
\hline $\begin{array}{l}\text { Zheng, S et } \\
\text { al }\end{array}$ & 2019 & circLRP6 & tissue & 50 & 50 & $\begin{array}{l}\text { qRT- } \\
\text { PCR }\end{array}$ & GAPDH & upregulated & 0.874 & [31] \\
\hline Zhu, $\mathrm{K}$ et al & 2019 & circ_0000885 & serum & 30 & 25 & $\begin{array}{l}\text { qRT- } \\
\text { PCR }\end{array}$ & $G A P D H$ & upregulated & 0.783 & [32] \\
\hline $\mathrm{Ma}, \mathrm{X}$ et al & 2018 & circ_HIPK3 & serum & 50 & 20 & $\begin{array}{l}\text { qRT- } \\
\text { PCR }\end{array}$ & GAPDH & downregulated & 0.783 & [24] \\
\hline $\mathrm{Xu}, \mathrm{B}$ et al & 2018 & CDR1as & tissue & 38 & 18 & $\begin{array}{l}\text { qRT- } \\
\text { PCR }\end{array}$ & $G A P D H$ & upregulated & 0.857 & [33] \\
\hline Zhou, $X$ et al & 2018 & circ_0008717 & tissue & 45 & 45 & $\begin{array}{l}\text { qRT- } \\
\text { PCR }\end{array}$ & GAPDH & upregulated & 0.782 & [34] \\
\hline $\begin{array}{l}\text { Zhu, K et al } \\
\text { (a) }\end{array}$ & 2018 & circPVT1 & serum & 50 & 20 & $\begin{array}{l}\text { qRT- } \\
\text { PCR }\end{array}$ & $G A P D H$ & upregulated & 0.871 & [19] \\
\hline $\begin{array}{l}\text { Zhu, K et al } \\
\text { (b) }\end{array}$ & 2018 & circ_0081001 & serum & 50 & 20 & $\begin{array}{l}\text { qRT- } \\
\text { PCR }\end{array}$ & GAPDH & upregulated & 0.898 & [18] \\
\hline Liu, $X$ et al & 2017 & circ-NT5C2 & tissue & 52 & 52 & $\begin{array}{l}\text { qRT- } \\
\text { PCR }\end{array}$ & $G A P D H$ & upregulated & 0.753 & [21] \\
\hline
\end{tabular}

Abbreviations: AUC, area under curve; CircRNA, circular RNA; qRT-PCR, quantitative real time polymerase chain reaction; Ref, reference 
Table 2

Main characteristics of the meta-analysis for prognosis association of circRNAs in osteosarcoma

\begin{tabular}{|c|c|c|c|c|c|c|c|c|c|c|c|}
\hline \multirow[t]{2}{*}{ Study } & \multirow[t]{2}{*}{$\begin{array}{l}\text { Publication } \\
\text { Year }\end{array}$} & \multirow[t]{2}{*}{ CircRNAs } & \multicolumn{2}{|c|}{$\begin{array}{l}\text { CircRNA } \\
\text { expression }\end{array}$} & \multirow[t]{2}{*}{$\begin{array}{l}\text { Sample } \\
\text { type }\end{array}$} & \multirow{2}{*}{$\begin{array}{l}\text { Detection } \\
\text { assay }\end{array}$} & \multirow[t]{2}{*}{$\begin{array}{l}\text { Regulation } \\
\text { pattern }\end{array}$} & \multirow[t]{2}{*}{$\begin{array}{l}\text { Survival } \\
\text { indicators }\end{array}$} & \multirow{2}{*}{$\begin{array}{l}\text { Survival } \\
\text { analysis }\end{array}$} & \multirow{2}{*}{$\begin{array}{l}\text { Follow- } \\
\text { up } \\
\text { months }\end{array}$} & \multirow[t]{2}{*}{ Ref. } \\
\hline & & & High & Low & & & & & & & \\
\hline $\begin{array}{l}\text { Wang, } \\
\text { L et al }\end{array}$ & 2020 & circ_0001658 & 21 & 18 & tissue & qRT-PCR & upregulated & N/A & N/A & N/A & [36] \\
\hline $\begin{array}{l}\mathrm{Li}, \mathrm{S} \text { et } \\
\mathrm{al}\end{array}$ & 2020 & circ_0000190 & 30 & 30 & tissue & qRT-PCR & downregulated & N/A & N/A & N/A & [30] \\
\hline $\begin{array}{l}\text { Wang, } \\
\text { Y et al }\end{array}$ & 2020 & circTCF25 & 26 & 24 & tissue & qRT-PCR & upregulated & N/A & N/A & N/A & [37] \\
\hline $\begin{array}{l}\mathrm{Ji}, \mathrm{X} \text { et } \\
\mathrm{al}\end{array}$ & 2020 & circ_001621 & 20 & 10 & tissue & qRT-PCR & upregulated & OS & Univariate & 60 & [12] \\
\hline $\begin{array}{l}\text { Zhu, K } \\
\text { et al }\end{array}$ & 2019 & circ_0000885 & 25 & 25 & tissue & qRT-PCR & upregulated & OS/DFS & Multivariate & 60 & [32] \\
\hline $\begin{array}{l}\text { Zheng, } \\
\text { S et al }\end{array}$ & 2019 & circLRP6 & N/A & N/A & tissue & qRT-PCR & upregulated & OS/DFS & Multivariate & 125 & [31] \\
\hline $\begin{array}{l}\text { Wang, } \\
\text { L et al }\end{array}$ & 2019 & circ_0021347 & 35 & 35 & tissue & qRT-PCR & downregulated & os & Univariate & 40 & [38] \\
\hline $\begin{array}{l}\text { Qi, H et } \\
\text { al }\end{array}$ & 2019 & circ_0000502 & 34 & 29 & tissue & qRT-PCR & upregulated & OS & Multivariate & 60 & [43] \\
\hline $\begin{array}{l}\text { Jin, J } \\
\text { et al }\end{array}$ & 2019 & circ_100876 & 24 & 24 & tissue & qRT-PCR & upregulated & OS & Univariate & 60 & [16] \\
\hline $\begin{array}{l}\text { Pan, G } \\
\text { et al }\end{array}$ & 2019 & circMMP9 & 24 & 27 & tissue & qRT-PCR & upregulated & OS & Univariate & 60 & [39] \\
\hline $\begin{array}{l}\mathrm{Li}, \mathrm{L} \text { et } \\
\mathrm{al}\end{array}$ & 2019 & circ_0001721 & 28 & 24 & tissue & qRT-PCR & upregulated & OS & Multivariate & 60 & [20] \\
\hline $\begin{array}{l}\text { Jin, Y } \\
\text { et al }\end{array}$ & 2019 & circ_0102049 & 38 & 38 & tissue & qRT-PCR & upregulated & OS & Multivariate & 60 & [17] \\
\hline $\begin{array}{l}\mathrm{Hu}, \mathrm{Y} \\
\text { et al }\end{array}$ & 2019 & circ-LARP4 & 36 & 36 & tissue & qRT-PCR & downregulated & OS/DFS & Univariate & 48 & [13] \\
\hline $\begin{array}{l}\text { Ma, X } \\
\text { et al }\end{array}$ & 2018 & circ_HIPK3 & 37 & 45 & tissue & qRT-PCR & downregulated & os & Univariate & 60 & [24] \\
\hline $\begin{array}{l}\text { Zhu, K } \\
\text { et al }\end{array}$ & 2018 & circPVT1 & 30 & 50 & tissue & qRT-PCR & upregulated & OS & Univariate & 60 & [19] \\
\hline $\begin{array}{l}\text { Zhu, K } \\
\text { et al }\end{array}$ & 2018 & circ_0081001 & 27 & 55 & tissue & qRT-PCR & upregulated & os & Multivariate & 60 & [18] \\
\hline $\begin{array}{l}\text { Zhu, K } \\
\text { et al }\end{array}$ & 2018 & circ_0004674 & 23 & 37 & tissue & qRT-PCR & upregulated & os & Univariate & 60 & [40] \\
\hline $\begin{array}{l}\text { Zhou, } \\
\text { X et al }\end{array}$ & 2018 & circ_0008717 & N/A & N/A & tissue & qRT-PCR & upregulated & OS/PFS & Multivariate & 80 & [34] \\
\hline $\begin{array}{l}\text { Huang, } \\
\text { L et al }\end{array}$ & 2018 & circNASP & 19 & 20 & tissue & qRT-PCR & upregulated & N/A & N/A & N/A & [14] \\
\hline $\begin{array}{l}\text { Nie, W } \\
\text { et al }\end{array}$ & 2018 & circ-NT5C2 & 86 & 84 & tissue & qRT-PCR & upregulated & OS/DFS & Multivariate & 60 & [41] \\
\hline $\begin{array}{l}\text { Li, B et } \\
\text { al }\end{array}$ & 2018 & circ_0007534 & 31 & 26 & tissue & qRT-PCR & upregulated & os & Multivariate & 60 & [23] \\
\hline $\begin{array}{l}\text { Wu, Z } \\
\text { et al }\end{array}$ & 2018 & circ_0002052 & 54 & 54 & tissue & qRT-PCR & downregulated & OS/PFS & Univariate & 60 & [35] \\
\hline $\begin{array}{l}\text { Zhang, } \\
\mathrm{H} \text { et al }\end{array}$ & 2018 & circ_001569 & 20 & 16 & tissue & qRT-PCR & upregulated & N/A & N/A & N/A & [22] \\
\hline $\begin{array}{l}\text { Zhang, } \\
\mathrm{H} \text { et al }\end{array}$ & 2017 & circUBAP2 & 42 & 50 & tissue & qRT-PCR & upregulated & os & Univariate & 60 & [42] \\
\hline
\end{tabular}


Abbreviations: CircRNA, circular RNA; DFS, disease-free survival; N/A, not available; OS, overall survival; PFS, progression-free survival; qRT-PCR, quantitative real time polymerase chain reaction; Ref, reference

The quality of studies was evaluated by QUADAS II and NOS scores. For diagnostic studies, the rating scores of QUADAS II ranged from 4 to 6. While for prognostic studies, the NOS scores were from 6 to 7, suggesting high methodological quality in all selected studies.

\section{Expression Of Circrna With Clinicopathological Parameters In Osteosarcoma}

The correlation between circRNAs and clinicopathological parameters in patients with osteosarcoma were demonstrated in Table 3. Overexpression of oncogenic circRNAs were significantly correlated with poor clinical features (tumor size: OR $=4.27,95 \% \mathrm{Cl}$ : 2.25-8.10; Enneking stage: OR = 5.52, 95\% Cl: 2.79-10.94; differentiation: $\mathrm{OR}=3.06,95 \% \mathrm{Cl}: 1.72-5.45$; DM: OR = 4.55, 95\% Cl: 2.55-8.12). In contrast, upregulated expression of tumorsuppressor circRNAs were remarkably associated with improved clinicopathological characteristics (Enneking stage: OR = 0.33, 95\% Cl: $0.16-0.68$; DM: $O R=0.18,95 \% \mathrm{Cl}: 0.08-0.40)$. Besides, no obvious difference was noted in terms of age and gender.

Table 3

Clinicopathological characteristics of circRNAs in osteosarcoma

\begin{tabular}{|c|c|c|c|c|c|c|}
\hline \multirow[t]{2}{*}{ Clinicopathological parameters } & \multicolumn{3}{|c|}{ Upregulated circRNAs } & \multicolumn{3}{|c|}{ Downregulated circRNAs } \\
\hline & OR & $95 \% \mathrm{Cl}$ & $p$ & OR & $95 \% \mathrm{Cl}$ & $p$ \\
\hline Age & 1.02 & $0.69-1.50$ & 0.93 & 0.94 & $0.47-1.87$ & 0.86 \\
\hline Gender (male/female) & 1.24 & $0.95-1.63$ & 0.12 & 1.01 & $0.59-1.74$ & 0.98 \\
\hline Tumor size $(\geq 5 \mathrm{~cm} /<5 \mathrm{~cm})$ & 4.27 & $2.25-8.10$ & $<0.00001$ & $\mathrm{~N} / \mathrm{A}$ & & \\
\hline Enneking stage (IIB-III /I-IIA) & 5.52 & $2.79-10.94$ & $<0.00001$ & 0.33 & $0.16-0.68$ & 0.002 \\
\hline WHO grade (III/ I-II) & 3.06 & $1.72-5.45$ & 0.0001 & $\mathrm{~N} / \mathrm{A}$ & & \\
\hline Distant metastasis $(\mathrm{P} / \mathrm{N})$ & 4.55 & $2.55-8.12$ & $<0.00001$ & 0.18 & $0.08-0.40$ & $<0.0001$ \\
\hline
\end{tabular}

Abbreviations: $\mathrm{Cl}$, confidence interval; $\mathrm{N}$, negative; N/A, not available; OR, odds ratio; $\mathrm{P}$, positive

\section{Diagnosis Analysis}

As demonstrated in Fig. 2, the forest plots showed that the pooled diagnostic value of circRNA in separating osteosarcoma from controls were as follows: sensitivity (SENS) of 0.80 (95\% Cl: 0.74-0.84), specificity (SPEC) of 0.80 (95\% Cl: 0.75-0.84), positive likelihood ratio (PLR) of 3.95 (95\% Cl: 3.19-4.89), negative likelihood ratio (NLR) of 0.26 (95\% Cl: $0.20-0.33$ ) and combined diagnostic odds ratio (DOR) of 15.48 (95\% Cl: $10.85-$ 22.10) (Fig. 2A, 2B, and 2C). Moreover, a summary receiver operator characteristic (SROC) curve was presented in Fig. 2D, and the AUC was 0.86 (95\% Cl: 0.83-0.89). These results indicated that circRNAs could be ideal diagnostic biomarker for osteosarcoma.

Additionally, a small set of oncogenic circRNAs with different diagnosis efficiency were synthesized in order to explore the combination panel of certain circRNAs with even higher diagnostic potential, which may facilitate the clinical application of the circRNAs for diagnosis. Among them, the pooled analysis containing circPVT1, circ_0081001, and CDR1as, has the highest diagnostic efficiency [18, 19, 33]. As shown in Figure S1, the forest plot of the pooled DOR, SENS and SPEC of these three circRNAs panel were 32.21 (95\%: 9.51-109.1), 0.85 (95\% Cl: 0.78-0.90), and 0.83 (95\% Cl: 0.71-0.91), respectively. Notably, the AUC under SROC curve was 0.9116 , indicating the combination of these circRNAs may have good diagnostic performance as the full panel of included circRNAs in osteosarcoma. However, more caution should be taken in the interpretation of this result since the comparatively small study numbers and limited sample size may produce outlying outcome and thereby introduce potential bias.

\section{Expression Of Circrnas With Prognosis In Osteosarcoma}

Survival analysis showed that overexpression of oncogenic circRNAs was significantly correlated with worse OS (HR = 1.92, 95\% Cl: $1.68-2.19)$, and DFS (HR $=2.65,95 \% \mathrm{Cl}: 2.02-3.49)$ as shown in Fig. 3A and 3B, respectively. We identified one outlier study performed by Zheng $S$ et al. in the combined effect of oncogenic circRNAs by sensitivity analysis. Besides, elevated expression of tumor-suppressor circRNAs predicted favorable OS $(\mathrm{HR}=0.44,95 \% \mathrm{Cl}: 0.28-0.69)$, as depicted in Fig. 3C. The fixed-effect model was applied in these studies since no obvious heterogeneity was noted.

\section{Sensitivity Analysis And Publication Bias}

Page 6/13 
The sensitivity analysis was performed in the prognostic effect sizes by omitting the enrolled studies one by one. For pooled effects of upregulated circRNAs in osteosarcoma, one study conducted by Zheng $S$ et al. [31] was identified as the outlier (Fig. 4A). Notably, the predictive significance of OS for upregulated circRNA did not alter after excluding the aforementioned outlier data $(\mathrm{HR}=2.51,95 \% \mathrm{Cl}$ : 2.08-3.02). No outliers were found in other pooled effects (Fig. 4B, 4C).

The Deeks' funnel plot test $(P=0.27)$ demonstrated the absence of publication bias existed in diagnostic analysis (Fig. $5 \mathrm{~A})$. For prognosis analysis of upregulated circRNA on DFS and downregulated circRNA on OS, the Begg's funnel plot also showed symmetry and revealed no evidence of publication bias among the eligible studies (Fig. 5C, 5D). Nevertheless, the funnel plot of upregulated circRNA profile on OS showed significant asymmetry, indicating a possible publication bias (Fig. 5B). Therefore, a "trim and fill" method was applied to trace the possible impacts from bias as previously described. However, the predictive value of upregulated circRNAs on OS was not altered after adjustment, suggesting a closely correlation between upregulated circRNAs and poor OS among osteosarcoma patients (Fig. 5C).

\section{Discussion}

Emerging studies have established an important role of circRNAs in cancer initiation and progression. In recent years, circRNAs have been recommended as novel diagnostic and prognostic biomarkers in several cancers [44], including colorectal cancer [29], hepatocellular cancer [45, 46], gastric cancer [47], esophageal cancer [48], and lung cancer [9, 49]. Our study implicated a marked correlation between abnormal circRNAs expression levels with clinical, diagnostic and prognostic significance in osteosarcoma. In particular, oncogenic circRNAs with higher expression pattern were strikingly associated with unfavorable clinical features, including larger tumor size, advanced clinical stage, poor differentiation and DM, while the tumor-suppressive circRNAs showed an opposite correlation. For diagnosis roles, our study suggested the AUC of 0.86 , with $80 \%$ sensitivity and $80 \%$ specificity of circRNAs in osteosarcoma. Besides, for the prognostic value, abnormal expression of circRNAs was remarkably related with OS as well as DFS. Furthermore, there were two studies reported the correlation between circRNA levels with PFS. Specifically, one demonstrated that highly expressed circ_0008717 was associated with worse PDS in osteosarcoma patients [34], while another study conducted by Wu Z et al. [35] showed that downregulated circ_0002052 predicted unfavorable PFS. Given the discrepant expression pattern of these two circRNA, it was unable to perform a pooled analysis concerning the prognostic significance of circRNA on PFS. Therefore, more studies describing the relationship between circRNAs and PFS in osteosarcoma are still needed.

Previously, Huang X et al. performed a meta-analysis on predictive values of circRNAs in osteosarcoma in 2019 [15]. However, only 13 studies were enrolled in their study, with 9 about clinical features, 11 on prognosis and 5 about diagnosis. Therefore, the pooled resulted may be underpowered due to insufficient data. Since the studies on circRNAs in osteosarcoma are emerging, we updated the predictive value of circRNAs in osteosarcoma in the study, with 26 studies comprising 1,652 patients, which markedly increased the statistical power and made the pooled results more credible. Besides, Huang $X$ and colleagues did not report the association between upregulated circRNA with DFS of osteosarcoma due to limited eligible studies, whereas our study report for the first time that oncogenic circRNA was significantly correlated with poor DFS in addition to OS.

The diagnostic value of circRNAs as potential biomarkers for osteosarcoma was extensively explored in our study. Aberrant expression of circRNAs was shown in a wide range of sample sources from osteosarcoma cell lines or patients, including tumor tissue and plasma. Given the fact that circRNA is widely expressed in human samples and stable in structure, it may be ideal biomarker candidate with diagnostic significance in osteosarcoma [50]. The SROC in our study showed that the pooled AUC of circRNA in osteosarcoma was 0.86 , indicating that $86 \%$ of randomly chosen osteosarcoma patients would have abnormal expression levels of circRNAs when compared with controls. Compared with receiver operating characteristic (ROC), which only compares single test accuracy over divergent thresholds for positivity, the each data point in SROC originates from a different study rather than a different threshold [51, 52]. Thus, SROC analysis could provide better evaluation for overall accuracy across SENS and SPEC from multiple studies [53]. Of note, an AUC under SROC curve over 0.75 usually indicates good diagnostic accuracy. Accordingly, the AUC of 0.86 suggests that our analysis was accurate and credible [53].

Moreover, the respective PLR and NLR were 3.95 and 0.26 , which indicated that the circRNA signature reached 3.95 between the true positive and false positive rates, and the probability of osteosarcoma patients that tested negative for circRNAs versus the case tested positive had a ratio of 0.26. In addition, the pooled DOR of 15.48 was obtained, implicating that dysregulated circRNAs were a powerful predictive biomarker for osteosarcoma diagnosis. Currently, serum alkaline phosphatase (ALP), with AUC of 0.673 , is one of the well-known biomarkers for osteosarcoma diagnosis [19]. Moreover, the sensitivity of ALP in OS diagnosis is also comparatively low, since over $40 \%$ of osteosarcoma patients may have normal ALP expression [3]. Our results indicated that dysregulated circRNAs may be even better than ALP for separating osteosarcoma patients from normal subjects with both enhanced sensitivity and specificity, suggesting a potential application in clinical practice.

However, several obstacles also remain in the utility of circRNAs for diagnosis. For instance, circRNAs obtained from tissue may be invasive to the patients, and detection of circRNAs from exosome may be expensive or technically difficult, which could limit the widespread application of multiple circRNAs as biomarkers [6]. Therefore, we also explored the diagnosis accuracy of the combination of a small set of certain oncogenic circRNAs, which may be more available than the full panel of included circRNAs in clinical application. Our preliminary pooled result showed that combination of three oncogenic circRNA, including circPVT1, circ_0081001, and CDR1as, has an AUC of 0.9116, with SENS of 0.85 and SPEC of 0.83 in 
diagnosis. However, as we mentioned above, the interpretation of the small set of circRNAs in separating osteosarcoma patients from control should be more cautious due to the small study number as well as limited sample size.

Additionally, it worth noted that several other limitations remain to be addressed in this study. First, the included number of article on downregulated circRNAs was still comparatively small, making it difficult to conduct a stratified analysis in terms of certain clinical characteristics. Second, some

HRs with $95 \%$ Cls were indirectly extracted from Kaplan-Meier curves, which may generate possible bias. Third, the detailed function and underlying mechanisms of circRNAs in osteosarcoma were not thoroughly annotated. Fourth, all of the studies were performed in China, which may introduce population bias. Thus, more studies with different ethnic groups are still warranted in future studies.

\section{Conclusion}

This study indicated a significant correlation between aberrant expression of circRNAs with the clinical, diagnostic and prognostic values in osteosarcoma patients. Therefore, circRNAs could serve as promising biomarkers and therapeutic targets for osteosarcoma.

\section{Abbreviations}

ALP: alkaline phosphatase; AUC:area under the curve; ceRNA:competing endogenous RNA; Cls:confidence intervals; CircRNAs:circular RNAs; DFS:disease-free survival; DM:distant metastasis; DOR:diagnostic odds ratio; HRs:hazard ratios; miRNA:microRNA; ncRNAs:non-coding RNAs; NLR:negative likelihood ratio; ORs:odds ratios; OS:overall survival; PFS:progression-free survival; PLR:positive likelihood ratio; PRISMA:Preferred Reporting Items for Systematic Review and Meta-analysis; QUADAS:Quality assessment for studies of diagnostic accuracy; RBP:RNA-binding protein; ROC:receiver operating characteristic; SENS:sensitivity; SPEC:specificity; SROC:summary receiver operator characteristic

\section{Declarations}

\section{Acknowledgements}

Not applicable.

\section{Authors' contributions}

Conception and design: CT; Collection and assembly of data: $\mathrm{CHZ}$ and JYH; Data analysis and interpretation: All authors; Manuscript writing: CT and ZHL; Final approval of manuscript: All authors.

\section{Funding}

This work was supported by the National Natural Science Foundation of China [81902745]; Natural Science Foundation of Hunan Province, China [2018JJ3716]; and China Scholarship Council [grant number [201806375067, 201806375068].

\section{Availability of data and materials}

The data used and analyzed in the study is available from the corresponding author on reasonable request.

\section{Ethics approval and consent to participate}

Not applicable.

\section{Consent for publication}

Not applicable.

\section{Competing Interest}

Chao Tu and Zhihong Li are members of the editorial board of BMC Cancer. Other authors declare that they approve this article and have no competing interests.

\section{References}

1. Li SQ, Tu C, Wan L, Chen RQ, Duan ZX, Ren XL, et al. FGF-induced LHX9 regulates the progression and metastasis of osteosarcoma via FRS2/TGF-beta/beta-catenin pathway. Cell Div. 2019;14:13.

2. Wang Z, Wu B, Zhou Y, Huang X, Pan W, Liu M, et al. Predictors of the Survival of Primary and Secondary Older Osteosarcoma Patients. J Cancer. 2019;10:4614-22. 
3. Cao LL, Chen Z, Yue Z, Pei L, Jia M, Wang H, et al. Novel classifiers with clinical laboratory parameters for early detection of osteosarcoma. J Clin Lab Anal. 2020: e23189.

4. Gupta M, Chandan K, Sarwat M. Role of miRNA and Long Non-Coding RNA in Hepatocellular Carcinoma. Curr Pharm Des. 2020;26:415-28.

5. Jahani S, Nazeri E, Majidzadeh AK, Jahani M, Esmaeili R. Circular RNA. a new biomarker for breast cancer: A systematic review. J Cell Physiol. 2020;235:5501-10.

6. Meng S, Zhou H, Feng Z, Xu Z, Tang Y, Li P, et al. CircRNA: functions and properties of a novel potential biomarker for cancer. Mol Cancer. 2017;16:94.

7. Zhang M, Huang N, Yang X, Luo J, Yan S, Xiao F, et al. A novel protein encoded by the circular form of the SHPRH gene suppresses glioma tumorigenesis. Oncogene. 2018;37:1805-14.

8. Zheng X, Chen L, Zhou Y, Wang Q, Zheng Z, Xu B, et al. A novel protein encoded by a circular RNA circPPP1R12A promotes tumor pathogenesis and metastasis of colon cancer via Hippo-YAP signaling. Mol Cancer. 2019;18:47.

9. Huang X, Zhang W, Shao Z. Prognostic and diagnostic significance of circRNAs expression in lung cancer. J Cell Physiol. 2019;234:18459-65.

10. Yin Y, Long J, He Q, Li Y, Liao Y, He P, et al. Emerging roles of circRNA in formation and progression of cancer. J Cancer. 2019;10:5015-21.

11. Fang X, Wen J, Sun M, Yuan Y, Xu Q. CircRNAs and its relationship with gastric cancer. J Cancer. 2019;10:6105-13.

12. Ji X, Shan L, Shen P, He M. Circular RNA circ_001621 promotes osteosarcoma cells proliferation and migration by sponging miR-578 and regulating VEGF expression. Cell Death Dis. 2020;11:18.

13. Hu Y, Gu J, Shen H, Shao T, Li S, Wang W, et al. Circular RNA LARP4 correlates with decreased Enneking stage, better histological response, and prolonged survival profiles, and it elevates chemosensitivity to cisplatin and doxorubicin via sponging microRNA-424 in osteosarcoma. $\mathrm{J}$ Clin Lab Anal. 2020;34:e23045.

14. Huang L, Chen M, Pan J, Yu W. Circular RNA circNASP modulates the malignant behaviors in osteosarcoma via miR-1253/FOXF1 pathway. Biochem Biophys Res Commun. 2018;500:511-7.

15. Huang X, Yang W, Zhang Z, Shao Z. Dysregulated circRNAs serve as prognostic and diagnostic markers in osteosarcoma by sponging microRNA to regulate the downstream signaling pathway. J Cell Biochem. 2020;121:1834-41.

16. Jin J, Chen A, Qiu W, Chen Y, Li Q, Zhou X, et al. Dysregulated circRNA_100876 suppresses proliferation of osteosarcoma cancer cells by targeting microRNA-136. J Cell Biochem. 2019;120:15678-87.

17. Jin Y, Li L, Zhu T, Liu G. Circular RNA circ_0102049 promotes cell progression as ceRNA to target MDM2 via sponging miR-1304-5p in osteosarcoma. Pathol Res Pract. 2019;215:152688.

18. Kun-Peng Z, Chun-Lin Z, Jian-Ping H, Lei Z. A novel circulating hsa_circ_0081001 act as a potential biomarker for diagnosis and prognosis of osteosarcoma. Int J Biol Sci. 2018;14:1513-20.

19. Kun-Peng Z, Xiao-Long M, Chun-Lin Z. Overexpressed circPVT1, a potential new circular RNA biomarker, contributes to doxorubicin and cisplatin resistance of osteosarcoma cells by regulating ABCB1. Int J Biol Sci. 2018;14:321-30.

20. Li L, Guo L, Yin G, Yu G, Zhao Y, Pan Y. Upregulation of circular RNA circ_0001721 predicts unfavorable prognosis in osteosarcoma and facilitates cell progression via sponging miR-569 and miR-599. Biomed Pharmacother. 2019;109:226-32.

21. Liu X, Zhong Y, Li J, Shan A. Circular RNA circ-NT5C2 acts as an oncogene in osteosarcoma proliferation and metastasis through targeting miR448. Oncotarget. 2017;8:114829-38.

22. Zhang H, Yan J, Lang X, Zhuang Y. Expression of circ_001569 is upregulated in osteosarcoma and promotes cell proliferation and cisplatin resistance by activating the Wnt/beta-catenin signaling pathway. Oncol Lett. 2018;16:5856-62.

23. Li B, Li X. Overexpression of hsa_circ_0007534 predicts unfavorable prognosis for osteosarcoma and regulates cell growth and apoptosis by affecting AKT/GSK-3beta signaling pathway. Biomed Pharmacother. 2018;107:860-6.

24. Xiao-Long M, Kun-Peng Z, Chun-Lin Z. Circular RNA. circ_HIPK3 is down-regulated and suppresses cell proliferation, migration and invasion in osteosarcoma. J Cancer. 2018;9:1856-62.

25. Liberati A, Altman DG, Tetzlaff J, Mulrow C, Gotzsche PC, loannidis JP, et al. The PRISMA statement for reporting systematic reviews and metaanalyses of studies that evaluate health care interventions: explanation and elaboration. J Clin Epidemiol. 2009;62:e1-34.

26. Whiting PF, Rutjes AW, Westwood ME, Mallett S, Deeks JJ, Reitsma JB, et al. QUADAS-2: a revised tool for the quality assessment of diagnostic accuracy studies. Ann Intern Med. 2011;155:529-36.

27. Zhang C, Ren X, He J, Wang W, Tu C, Li Z. The prognostic value of long noncoding RNA SNHG16 on clinical outcomes in human cancers: a systematic review and meta-analysis. Cancer Cell Int. 2019;19:261.

28. Tu C, Ren X, He J, Zhang C, Chen R, Wang W, et al. The Value of LncRNA BCAR4 as a Prognostic Biomarker on Clinical Outcomes in Human Cancers. J Cancer. 2019;10:5992-6002.

29. Li C, He X, Zhang L, Li L, Zhao W. A pair-wise meta-analysis highlights circular RNAs as potential biomarkers for colorectal cancer. BMC Cancer. 2019;19:957.

Page 9/13 
30. Li S, Pei Y, Wang W, Liu F, Zheng K, Zhang X. Extracellular nanovesicles-transmitted circular RNA has_circ_0000190 suppresses osteosarcoma progression. J Cell Mol Med. 2020;24:2202-14.

31. Zheng S, Qian Z, Jiang F, Ge D, Tang J, Chen H, et al. CircRNA LRP6 promotes the development of osteosarcoma via negatively regulating KLF2 and APC levels. Am J Transl Res. 2019;11:4126-38.

32. Zhu K, Niu L, Wang J, Wang Y, Zhou J, Wang F, et al. Circular RNA hsa_circ_0000885 Levels are Increased in Tissue and Serum Samples from Patients with Osteosarcoma. Med Sci Monit. 2019;25:1499-505.

33. Xu B, Yang T, Wang Z, Zhang Y, Liu S, Shen M. CircRNA CDR1as/miR-7 signals promote tumor growth of osteosarcoma with a potential therapeutic and diagnostic value. Cancer Manag Res. 2018;10:4871-80.

34. Zhou X, Natino D, Qin Z, Wang D, Tian Z, Cai X, et al. Identification and functional characterization of circRNA-0008717 as an oncogene in osteosarcoma through sponging miR-203. Oncotarget. 2018;9:22288-300.

35. Wu Z, Shi W, Jiang C. Overexpressing circular RNA hsa_circ_0002052 impairs osteosarcoma progression via inhibiting Wnt/beta-catenin pathway by regulating miR-1205/APC2 axis. Biochem Biophys Res Commun. 2018;502:465-71.

36. Wang L, Wang P, Su X, Zhao B. Circ_0001658 promotes the proliferation and metastasis of osteosarcoma cells via regulating miR-382-5p/YB-1 axis. Cell Biochem Funct. 2020;38:77-86.

37. Wang Y, Shi S, Zhang Q, Dong H, Zhang J. MicroRNA-206 upregulation relieves circTCF25-induced osteosarcoma cell proliferation and migration. J Cell Physiol. 2020; Feb 13.

38. Wang L, Zhang GC, Kang FB, Zhang L, Zhang YZ. hsa_circ0021347 as a Potential Target Regulated by B7-H3 in Modulating the Malignant Characteristics of Osteosarcoma. Biomed Res Int. 2019; 2019: 9301989.

39. Pan G, Hu T, Chen X, Zhang C. Upregulation Of circMMP9 Promotes Osteosarcoma Progression Via Targeting miR-1265/CHI3L1 Axis. Cancer Manag Res. 2019;11:9225-31.

40. Kun-Peng Z, Xiao-Long M, Lei Z, Chun-Lin Z, Jian-Ping H, Tai-Cheng Z. Screening circular RNA related to chemotherapeutic resistance in osteosarcoma by RNA sequencing. Epigenomics. 2018;10:1327-46.

41. Nie WB, Zhao LM, Guo R, Wang MX, Ye FG. Circular RNA circ-NT5C2 acts as a potential novel biomarker for prognosis of osteosarcoma. Eur Rev Med Pharmacol Sci. 2018;22:6239-44.

42. Zhang H, Wang G, Ding C, Liu P, Wang R, Ding W, et al. Increased circular RNA UBAP2 acts as a sponge of miR-143 to promote osteosarcoma progression. Oncotarget. 2017;8:61687-97.

43. Qi H, Sun Y, Jiang Y, Li X. Upregulation of circular RNA circ_0000502 predicts unfavorable prognosis in osteosarcoma and facilitates cell progression via sponging miR-1238. J Cell Biochem. 2018; Dec 7.

44. Tan H, Gan L, Fan X, Liu L, Liu S. Diagnostic value of circular RNAs as effective biomarkers for cancer: a systematic review and meta-analysis. Onco Targets Ther. 2019;12:2623-33.

45. Jiang YL, Shang MM, Dong SZ, Chang YC. Abnormally expressed circular RNAs as novel non-invasive biomarkers for hepatocellular carcinoma: A meta-analysis. World J Gastrointest Oncol. 2019;11:909-24.

46. Huang X, Zhang W, Shao Z. Prognostic and diagnostic significance of circRNAs expression in hepatocellular carcinoma patients: A metaanalysis. Cancer Med. 2019;8:1148-56.

47. Chen H, Wang K, Pei D, Xu H. Appraising circular RNAs as novel biomarkers for the diagnosis and prognosis of gastric cancer: A pair-wise metaanalysis. J Clin Lab Anal. 2020: e23303.

48. Niu C, Zhao L, Guo X, Shen Y, Shao Y, Liu F. Diagnostic Accuracy of circRNAs in Esophageal Cancer: A Meta-Analysis. Dis Markers. 2019; 2019: 9673129.

49. Xiao Z, Chen X, Lu X, Zhong X, Ling Y. Accuracy Evaluation of Circular RNA in Diagnosing Lung Cancer in a Chinese Population. Dis Markers. 2019; 2019: 7485389.

50. Wang M, Yang Y, Xu J, Bai W, Ren X, Wu H. CircRNAs as biomarkers of cancer: a meta-analysis. BMC Cancer. 2018;18:303.

51. Vamvakas EC. Meta-analyses of studies of the diagnostic accuracy of laboratory tests: a review of the concepts and methods. Arch Pathol Lab Med. 1998;122:675-86.

52. Littenberg B, Moses LE. Estimating diagnostic accuracy from multiple conflicting reports: a new meta-analytic method. Med Decis Making. 1993;13:313-21.

53. Jones CM, Athanasiou T. Summary receiver operating characteristic curve analysis techniques in the evaluation of diagnostic tests. Ann Thorac Surg. 2005;79:16-20.

\section{Figures}




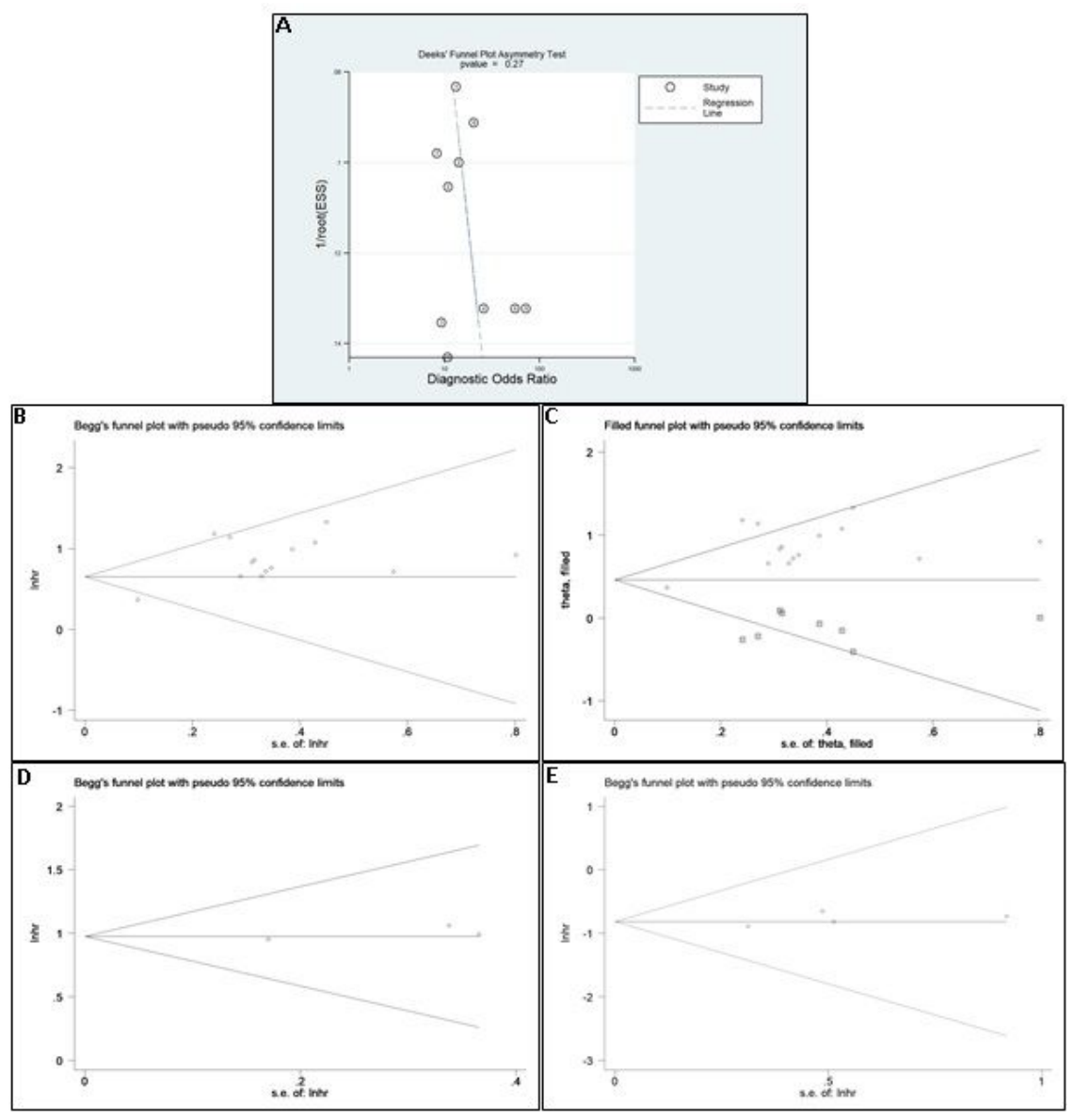

Figure 1

Publication bias judged by the funnel plots for diagnostic and prognostic analysis. Deek's funnel plot for the diagnostic effect (A). Begg's test for upregulated circRNA signature in predicting OS (B) and DFS (D), and downregulated circRNA in predicting OS (E) in osteosarcoma. The "trim and fill" method was applied to assess the possible effects of bias on the overall pooled effects of upregulated circRNA signature (C). The squares indicate the imputed studies. OS, overall survival; DFS, disease-free survival.

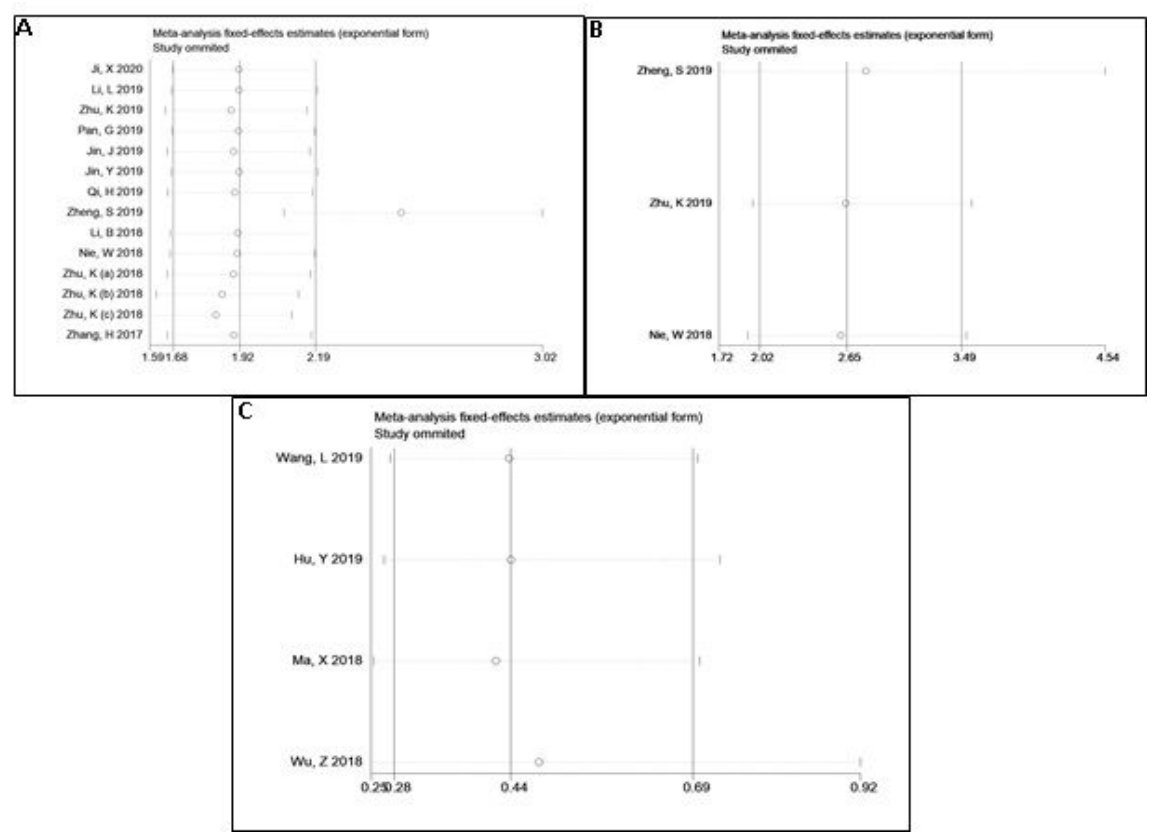

Figure 2

Sensitivity analysis of the upregulated circRNA profiles for OS (A) and DFS (B), and downregulated circRNA (C) signature for OS in osteosarcoma. OS, overall survival; DFS, disease-free survival. 

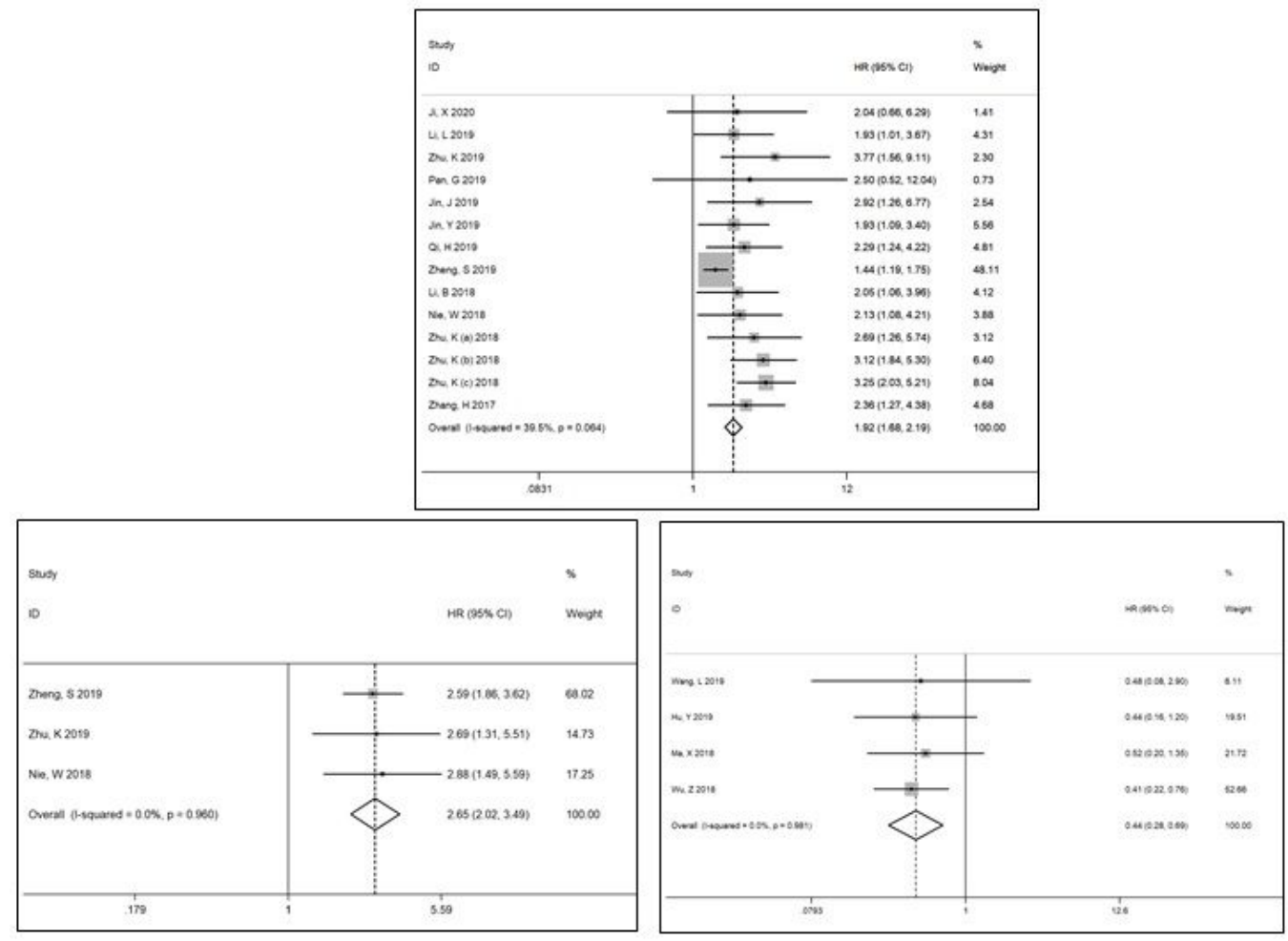

\section{Figure 3}

Forest plots describing the OS (A) and DFS (B) for the upregulated circRNAs, and OS (C) for downregulated circRNAs in osteosarcoma. OS, overall survival; DFS, disease-free survival.

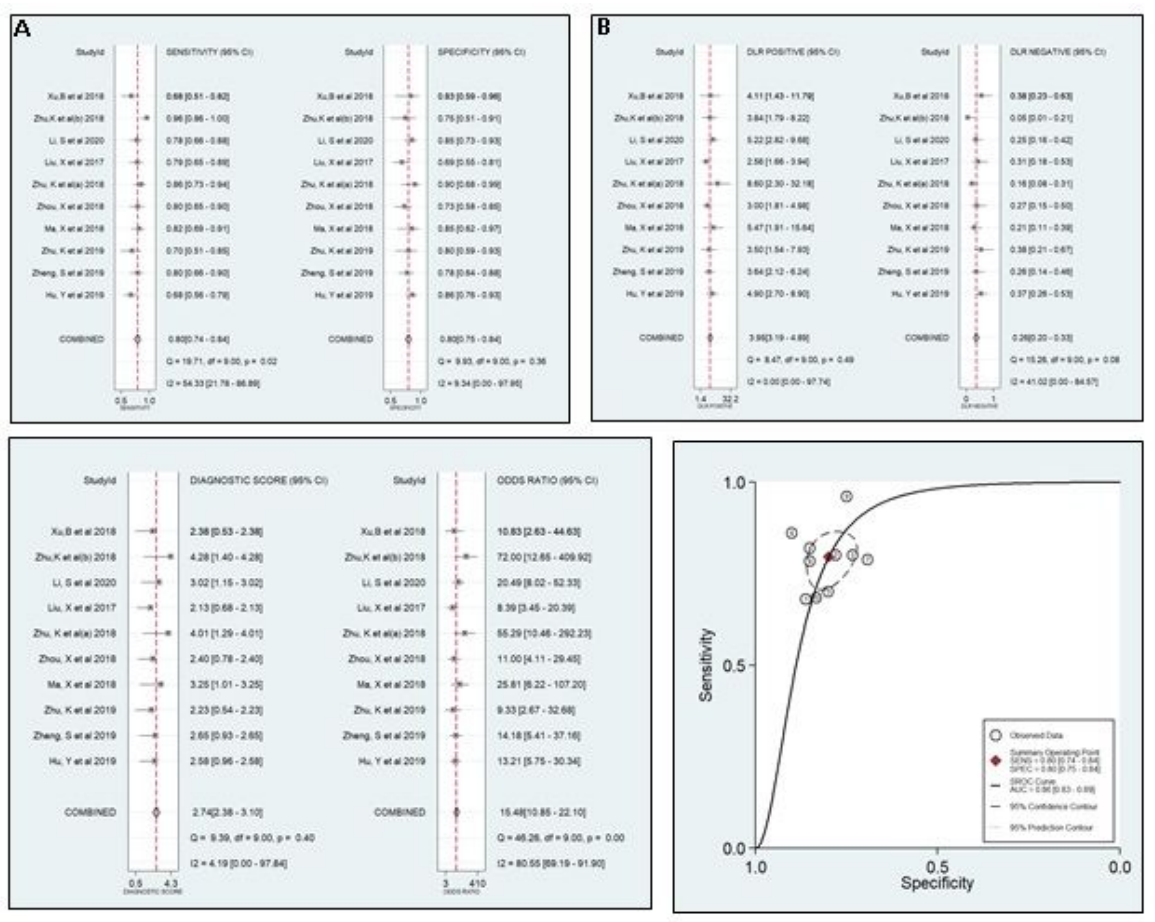

\section{Figure 4}

Forest plots of the combined sensitivity and specificity (A), PLR and NLR (B), DOR (C), and the SROC curve (D) in predicting diagnosis of osteosarcoma. DOR, diagnostic odds ratio; NLR, negative likelihood ratio; PLR, positive likelihood ratio; SROC, summary receiver operator characteristic. 
Records after duplicates removed

$$
(n=80)
$$
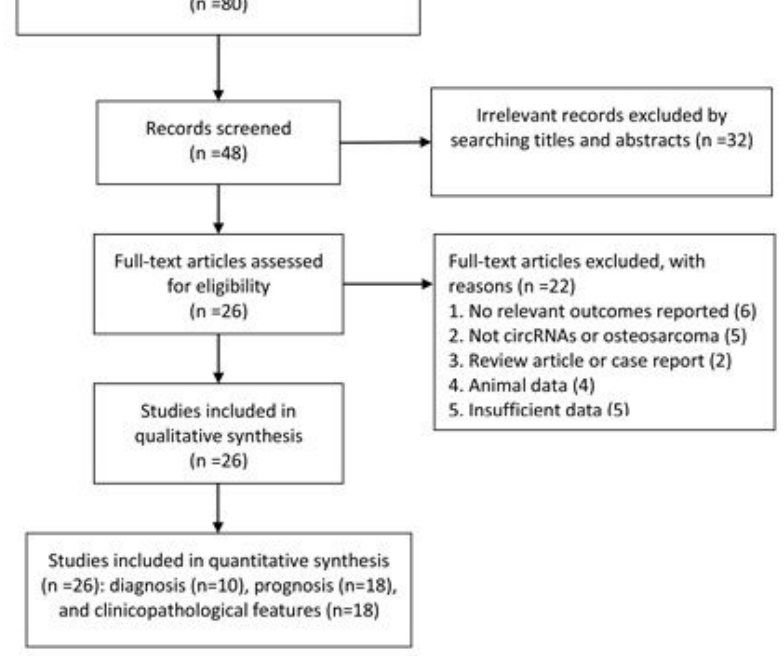

Figure 5

The PRISMA flow diagram of study selection process.

\section{Supplementary Files}

This is a list of supplementary files associated with this preprint. Click to download.

- Additionalfile1PRISMAChecklist.pdf 